\title{
Antioxidant Status in Elite Three-Day Event Horses during Competition
}

\author{
Carey A. Williams ${ }^{1}$ and Amy O. Burk ${ }^{2}$ \\ ${ }^{1}$ Department of Animal Sciences, Rutgers, The State University of New Jersey, New Brunswick, NJ 08901, USA \\ ${ }^{2}$ Animal and Avian Sciences Department, University of Maryland, College Park, MD 20742, USA
}

Correspondence should be addressed to Carey A. Williams, cwilliams@aesop.rutgers.edu

Received 14 February 2012; Accepted 22 April 2012

Academic Editor: Manfred Lamprecht

Copyright ( 2012 C. A. Williams and A. O. Burk. This is an open access article distributed under the Creative Commons Attribution License, which permits unrestricted use, distribution, and reproduction in any medium, provided the original work is properly cited.

\begin{abstract}
The objective of this study was to determine if competition intensity would have an effect on antioxidant status in horses before and during a three-day event. Body weight, body condition score, and blood was sampled from CCI2* $(n=19)$ and CCI3* $(n=23)$ horses before the start of dressage, 20 to $30 \mathrm{~min}$ following cross-country, and 18-24 h after cross-county. Data were analyzed using a PROC MIXED in SAS. There were no differences between CCI2* and CCI3* horses during competition for plasma cortisol, lactate, $\alpha$-tocopherol, retinol, or erythrocyte glutathione peroxidase. After cross-country, CCI3* horses had higher serum creatine kinase $(P=0.003)$ and aspartate aminotransferase $(P<0.0001)$ than the CCI $2 *$ horses. Plasma $\beta$-carotene was higher in the CCI2* horses compared to the CCI3* horses $(P=0.0001)$. Total erythrocyte glutathione was also higher in the CCI2* horses versus CCI3* horses $(P<0.0001)$. These results are the first report of antioxidant status of horses competing in this level of a three-day event. The changes in antioxidant and muscle enzymes observed between divisions are likely due to the increased anaerobic and musculoskeletal demand on the upper level horses and the fitness required to compete at that level.
\end{abstract}

\section{Introduction}

Equine athletes competing at the top levels of their disciplines experience physiological stress that may compromise health and performance. Oxidative stress results in oxidative damage to all cell components. Oxidative stress is caused by an imbalance between the reactive oxygen species (ROS) produced during cellular respiration and the body's antioxidant system used to scavenge these ROS [1]. Oxidative stress has been observed in horses exposed to intense bouts of exercise [2,3] and endurance exercise [4, 5]. Antioxidants are vitamins, minerals, and enzymes that must be synthesized in the body or obtained from the diet. Therefore, exercise level and diet are both factors that play a role in influencing the oxidative stress and antioxidant status of the equine athlete.

Eventing is a rigorous exercise challenge, similar to the human triathlon that tests the physical ability and skill of both horse and rider during three separate phases. In dressage, a standard set of movements is performed and awarded a subjective score. Cross-country jumping requires horses to jump over 35 to 40 obstacles covering 5 to $7 \mathrm{~km}$ of terrain within a limited time. For stadium, jumping horses are jumped over 10 to 12 obstacles arranged in a course set in an enclosed arena. The International Federation for Equestrian Sports (FEI) governs the sport and has divided it into four levels of difficulty. The highest or Olympic level is denoted as Concours Complet Internationale Four-Star Event $\left(\mathrm{CCI} 4^{*}\right)$ followed by the CCI3*, CCI2*, and CCI1* divisions.

Many studies previously have researched electrolyte loss and fluid shifts, lactate response, and other biochemical measures of exercise intensity of horses performing in 3day event competitions. However, none have measured the antioxidant status along with their dietary profile. Therefore, the main objective of this study was to determine the effects of a rigorous, high-level three-day event on the antioxidant status of horses. Recognizing that diet can influence antioxidant status, a second objective was to characterize the diet and feeding management practices of three-day event horses prior to and during the exercise challenge. It is hypothesized 
TABLE 1: Specifications of the cross-country jumping phase in the CCI2* and CCI $3 *$ divisions of the 2006 Jersey fresh three-day event.

\begin{tabular}{lcc}
\hline & CCI2* & CCI3* \\
\hline Distance & $4.4-5 \mathrm{~km}$ & $5.7-6.8 \mathrm{~km}$ \\
Speed & $9.2 \mathrm{~m} / \mathrm{s}$ & $9.5 \mathrm{~m} / \mathrm{s}$ \\
No. of jumps & 36 & 40 \\
Max. height & $1.15 \mathrm{~m}$ & $1.2 \mathrm{~m}$ \\
Max. drop & $1.8 \mathrm{~m}$ & $2 \mathrm{~m}$ \\
\hline
\end{tabular}

that the oxidative stress markers would be higher after crosscountry jumping in both divisions, but the $\mathrm{CCI} 3{ }^{*}$ would be higher than the $\mathrm{CCI} 2 *$ due to the higher level of exercise intensity.

\section{Materials and Methods}

2.1. Competition. Horses and riders participating in this study were competing in either the CCI ${ }^{*}$ or $\mathrm{CCI} 3 *$ division of the Jersey fresh three-day event in Allentown, NJ from May 31 to June 4, 2006. The event consisted of a postarrival veterinary horse inspection on day 1, dressage on $\mathrm{d} 2$ or 3 , cross-country jumping followed by the second veterinary horse inspection on $\mathrm{d} 4$, and then a third veterinary horse inspection followed by stadium jumping on $\mathrm{d} 5$. Competitors rode the 2005 FEI CCI2* or CCI3* event dressage test $\mathrm{B}$ for their respective divisions. The distance, optimum speed and jumping efforts for the cross-country jumping phase are presented in Table 1. Mean ambient temperature during the five days of competition was $22.9^{\circ} \mathrm{C}$ (range: 16.1 to $33.3^{\circ} \mathrm{C}$ ) with $5.4,3.3$, and $0.36 \mathrm{~cm}$ of precipitation occurring on days 3, 4, and 5 (Weather Data Services, Inc, Wichita, KS).

2.2. Subjects. Prior to the first veterinary horse inspection, horse owners or riders were asked to complete a release statement that voluntarily enrolled their horses as participants in the study. Nineteen of $33(58 \%)$ CCI ${ }^{*}$ horses and 23 out of $41(56 \%)$ CCI3 ${ }^{*}$ horses were enrolled. Horses in the CCI2* division completed dressage from 0900 to 1200 on days 2 and 3 of the competition and cross-country jumping between 0900 and 1100 on days 4. Horses in the $\mathrm{CCI}^{*}$ division completed dressage from 1300 to 1800 on $\mathrm{d}$ 2 and 3 and cross-country jumping between 1300 and 1600 on days 4. All stadium jumping started at 1200 on days 5 with CCI ${ }^{*}$ horses competing first, immediately followed by $\mathrm{CCI}^{*}$ horses.

2.3. Sample Collection and Processing. Heart rate, rectal temperature, and respiratory rate were recorded by a licensed veterinarian immediately following completion of the crosscountry jumping phase. Whole blood was collected on $\mathrm{d} 1$ immediately after the first veterinary horse inspection (PRE), on d 4 20-30 min following the cross-country jumping phase as part of the second veterinary horse inspection (XC), and on d 5 immediately after the third veterinary horse inspection but prior to the commencement of stadium jumping (POST). The POST sample was therefore taken 18 to $24 \mathrm{~h}$ after XC. Body weight (BW) was determined using an electronic scale, and body condition score (BCS) [6] was analyzed at the time of blood collection. Whole blood $(40 \mathrm{~mL})$ was collected by venipuncture into sodium heparin, EDTA, and serum separator Vacutainer tubes (Becton Dickinson and Company, Franklin Lakes, NJ), immediately placed on ice, and then processed into red blood cell, plasma, or serum aliquots at an on-site laboratory within $30 \mathrm{~min}$ of collection using previously described methods [7]. All processed samples were stored on dry ice until transferred to $-80^{\circ} \mathrm{C}$, where they remained until analysis (within $24 \mathrm{~h}$ of sample collection).

2.4. Sample Analysis. Whole blood was analyzed for hematocrit (Hct) using a microhaematocrit technique (Spiracrit, Oxford Labware-Division of Sherwood Medical, St. Louis, $\mathrm{MO})$. Plasma was analyzed for total protein (TP) using refractometry. Plasma cortisol (CORT) was analyzed by radioimmunoassay (Diagnostic Products Corporation, Los Angeles, CA). Plasma lactate (LAC) was analyzed using YSI model no. 1500 lactate analyzer (YSI Inc., Yellowsprings, $\mathrm{OH})$. Serum was analyzed for creatine kinase $(\mathrm{CK})$ and aspartate aminotransferase (AST) using a VetTest 8008 analyzer (Model VT8008, IDEXX Laboratories Inc., Westbrook, $\mathrm{ME})$.

The antioxidants $\alpha$-tocopherol (TOC), retinol (RET), and $\beta$-carotene (BC) were analyzed by HPLC (Michigan State Diagnostic Laboratories, East Lansing, MI) by first running plasma samples through a precolumn followed by a reverse phase C-18 HPLC column eluted isocratically at $1.2 \mathrm{~mL} / \mathrm{min}$ with an injection volume of $50 \mathrm{uL}$. Absorbance was measured for TOC (292 nM), RET (325 nM), and BC (450 nM). Red blood cells were analyzed for total glutathione (GSH-T) and glutathione peroxidase (GPx). Methods for analysis of GSHT (Biotech GSH-420, kit \#21023; Oxis Health Products Inc., Portland, OR; interassay CV 7.0\%, intra-assay CV 5.6\%) and cellular GPx (Biotech GPx-340, kit \#21017; Oxis Health Products Inc., Portland, OR; interassay CV 4.2\%, intra-assay CV 5.0\%) were previously described [7].

2.5. Statistical Analysis. Data are summarized as mean $\pm \mathrm{SE}$ unless otherwise noted. Effects of division, sample time, and their interaction were evaluated on antioxidant and oxidative stress markers, along with performance parameters, using a repeated model ANOVA with sample as our repeated effect (PROC MIXED, SAS Inst Inc., version 9.1, Cary, NC). To further test for differences between competition divisions and sample times, Tukey-Kramer adjustment was used with significance determined using $P<0.05$. Associations between exercise and antioxidant variables were tested using Pearson's product-moment correlation. Horse within division was included in the model to test for significance; if insignificant, then it was removed from the model. Significance was determined using $P<0.05$.

\section{Results}

3.1. Horse Data. Demographic information, initial BW, and initial BCS of horses participating in the study are shown 
TABLE 2: Subject information for horses and riders competing in the CCI ${ }^{*}$ and $\mathrm{CCI}^{*}$ divisions of the 2006 Jersey fresh three-day event.

\begin{tabular}{|c|c|c|}
\hline \multirow{2}{*}{ Item } & \multicolumn{2}{|c|}{ Divisions } \\
\hline & CCI2* & CCI3* \\
\hline Subject participation $^{1}, \%$ & 57.5 & 56.1 \\
\hline Age, yr & $11.3 \pm 0.7$ & $11.3 \pm 0.5$ \\
\hline \multicolumn{3}{|l|}{ Sex of horse, $\%$} \\
\hline Male & 78.9 & 91.3 \\
\hline Female & 21.1 & 8.7 \\
\hline \multicolumn{3}{|l|}{ Breed of horse, $\%$} \\
\hline Thoroughbred & 78.9 & 59.1 \\
\hline Thoroughbred cross & 10.5 & 22.7 \\
\hline Warmblood & 5.3 & 9.1 \\
\hline Other & 5.3 & 9.1 \\
\hline Initial BW, kg & $529.2 \pm 7.7$ & $529.7 \pm 7.1$ \\
\hline Initial BCS & $5.2 \pm 0.1$ & $5.1 \pm 0.1$ \\
\hline
\end{tabular}

${ }^{1}$ CCI2 ${ }^{*}, n=19$ out of 33 entered; CCI ${ }^{*}, n=23$ out 41 entered.

in Table 2. There were no differences in age, initial BW, and initial BCS between $\mathrm{CCI} 2 *$ and ${ }^{*} \mathrm{CI} 3 *$ horses. For both divisions, the majority of horses were of the male gender and Thoroughbred breed. Mean age was $11.3 \pm 0.7$ and $11.3 \pm 0.5$ yrs for $\mathrm{CCI} 2 *$ and $\mathrm{CCI} 3 *$ horses, respectively. Horses competing in the CCI2* had been competing at that level for 0 to $1 \mathrm{yr}(50.0 \%), 1$ to $2 \mathrm{yr}(30.0 \%)$, or 3 to $5 \mathrm{yr}$ $(20.0 \%)$, whereas horses in the CCI ${ }^{*}$ had been competing at that level for 0 to $1 \mathrm{yr}(52.2 \%), 1$ to $2 \mathrm{yr}(26.1 \%)$, or 3 to $5 \mathrm{yr}(21.7 \%)$.

Twelve subjects completed the CCI $2 *(63.2 \%)$ with seven placing in the top 10 (1st, 2nd, 3rd, 6th, 8th, 9th, and 10th). Of the nonfinishers, three withdrew before cross-country jumping, three withdrew or were eliminated during crosscountry jumping, and one did not pass the final veterinary horse inspection. In the $\mathrm{CCI} 3^{*}, 13$ subjects completed the event $(56.5 \%)$ with four placing in the top 10 (3rd, 6th, 9th, and 10th). Nonfinishers included one that was withdrawn before cross-county jumping, six that were withdrawn or were eliminated during cross-county jumping, and three that were either withdrawn after cross-country jumping for unknown reasons or did not pass the final veterinary horse inspection.

Mean heart rate, rectal temperature, and respiratory rate immediately following the completion of cross-country jumping were not different between divisions (data not shown). For all horses completing the cross-country jumping phase, mean heart rate was $102 \pm 2.8$ beats per min, rectal temperature was $40.4 \pm 0.09^{\circ} \mathrm{C}$, and respiratory rate was $100 \pm 3.2$ breaths per min. There were no correlations observed between these variables and blood variables assessed at XC (data not shown).

3.2. Diet Data. Diets of horses could not be controlled in this study, and as a result, horses were fed according to the rider or owner's preference. A nutritional management survey was conducted prior to and during the competition to gather information on each horse's feeding times; type of pasture and hours spent turned out on pasture prior to competition; type and amount of hay, concentrate, and supplements offered at competition; feeding associated with transport to the event and cross-country jumping. All feeding management data collected during the study have been previously reported [8].

\subsection{Blood Data}

3.3.1. Effect of Division. There was no main effect of the competition division on plasma TP, CORT, LAC, AST, TOC, RET, BC, and erythrocyte GPx; therefore, data were averaged across division (Table 3 ). Figure 1 shows blood variables that differed between horses competing in the CCI $2^{*}$ and $\mathrm{CCI}^{*}$ divisions. There was a main effect of division on Hct $(P=0.002)$, and erythrocyte GSH-T $(P<0.0001)$ with the CCI2* horses having higher blood concentrations than the $\mathrm{CCI}^{*}$ horses. For serum CK $(P=0.04)$, however, serum concentrations were lower in the CCI2* horses compared to the $\mathrm{CCI}^{*}{ }^{*}$ horses. For Hct, $\mathrm{CCI} 2 *$ horses were higher at $\mathrm{XC}$ than $\mathrm{CCI}^{*}$ horses $(P=0.001 ; 55.1 \pm 1.7$ versus $50.1 \pm 1.0 \%$, resp.; Figure $1(\mathrm{a}))$. Serum CK was lower in CCI2* horses at $\mathrm{XC}$ than $\mathrm{CCI}^{*}$ horses $(P=0.003 ; 235 \pm 16$ versus $576 \pm 123 \mathrm{mmol} / \mathrm{L}$; Figure $1(\mathrm{~b}))$. Red blood cell GSH-T was higher in CCI2* horses at XC $(P<0.0001 ; 237 \pm 12$ versus $163 \pm 12 \mathrm{uM}$, resp.) compared to $\mathrm{CCI}^{*}$ horses (Figure $1(\mathrm{c})$ ), and a time by division interaction was observed $(P=0.006)$.

3.3.2. Effect of Time. The Hct was highest at the XC sample for all horses $(P<0.0001$, Figure $1(\mathrm{a}))$, but was not different between PRE and POST. Plasma TP was different for all three samples $(P<0.0003$, Table 3$)$ with the highest occurring at $\mathrm{XC}$. Plasma CORT was highest after XC and remained higher than PRE levels at POST $(P<0.005$, Table 3$)$. Serum CK, AST, and plasma LAC were all lowest at PRE $(P<0.01$, Figure 1(b), and Table 3, resp.). Plasma TOC did not change in horses throughout the competition (Table 3). Plasma BC was highest in horses at $\operatorname{PRE}(P=0.003$; Table 3$)$ and decreased to XC and POST. Plasma RET increased in all horses from PRE to XC, but it then decreased to its lowest value at POST $(P<0.03$, Table 3$)$. Red blood cell GSH-T was similar from PRE to XC, but it then decreased below baseline at POST $(P<0.0001$, Figure $1(\mathrm{c}))$. Red blood cell GPx peaked at XC compared to PRE and POST $(P=0.004$, Table 3).

3.3.3. Correlations. Significant correlations between the exercise and antioxidant variables are shown in Table 4. Some of these include a positive correlation between LAC and GSHT, GPx, CORT and RET $(P<0.05)$. Plasma CORT, was also positively correlated with $\mathrm{CK}(P=0.007)$, RET $(P=0.003)$, and GSH-T $(P=0.04)$ and negatively correlated with TOC $(P=0.04)$. Plasma AST, however, was not correlated with CORT as CK was, but was positively correlated with TOC $(P=0.002)$ and negatively correlated with GPx $(P=0.04)$. 


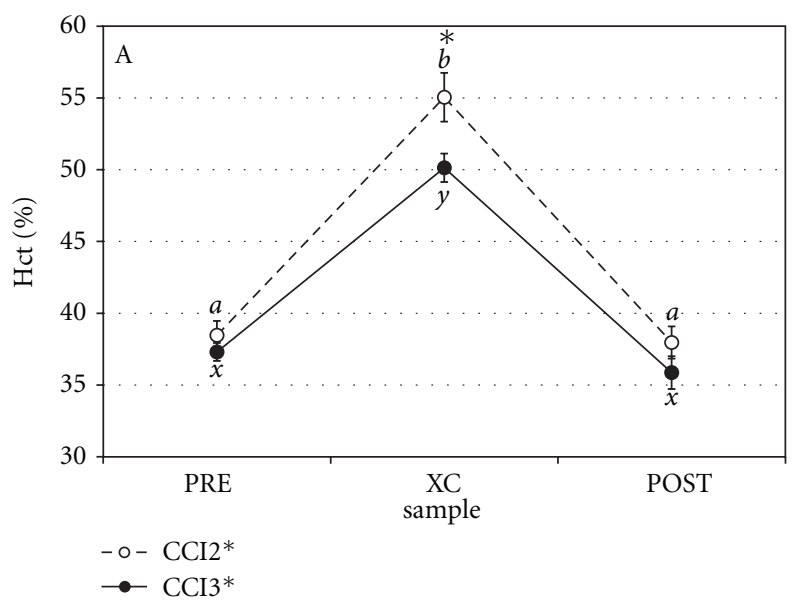

(a)

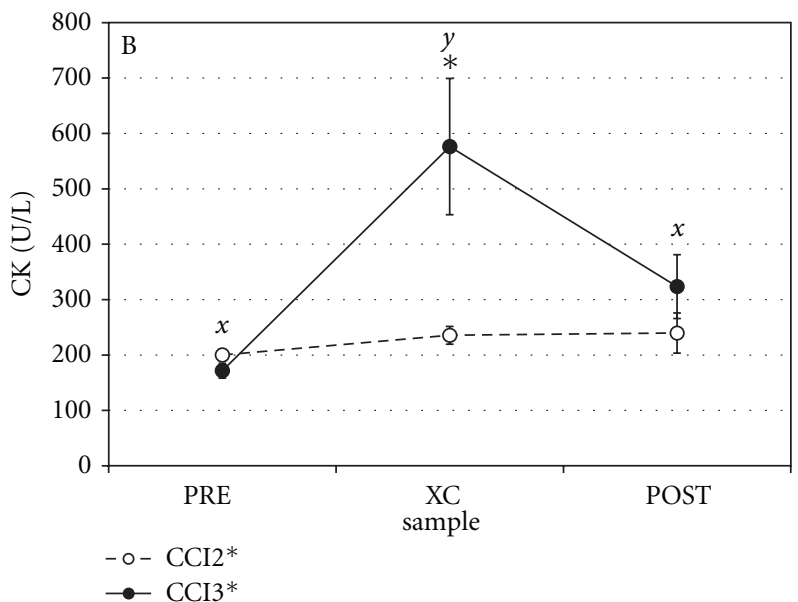

(b)

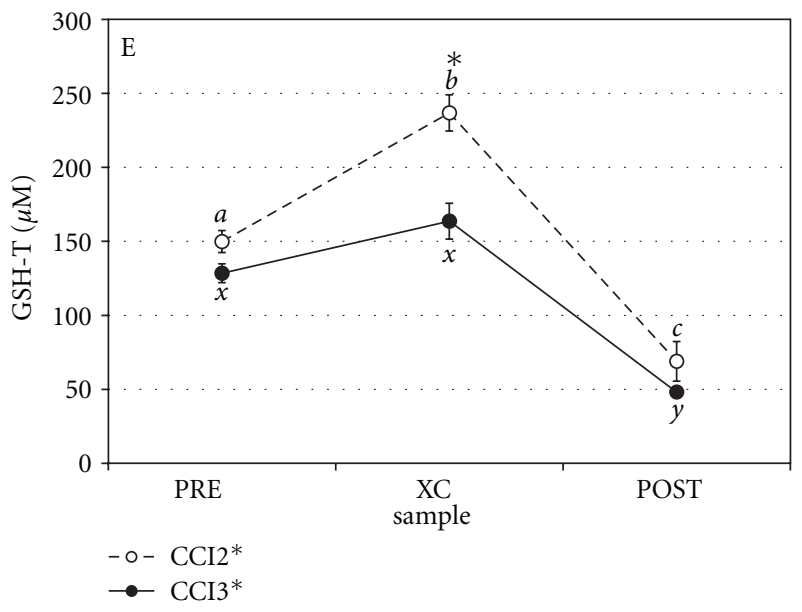

(c)

FIgURE 1: Hematocrit (Hct; (a)), serum creatine kinase (CK; (b)), and red blood cell total glutathione (GSH-T; (c)) in CCI2* and CCI3* horses before competition (PRE), 20-30 min after cross-country (XC), and 18-24 hr after XC but before stadium jumping (POST). * denotes difference between $\mathrm{CCI} 2 *$ and CCI $3 *$ horses within sampling time $(P<0.001)$. Letters a, b, and c denote differences across sampling time for CCI $2^{*}$ horses $(P<0.05)$. Letters $x, y$, and $z$ denote differences across sampling time for CCI $3 *$ horses $(P<0.05)$.

TABLE 3: Blood variables assessed before the competition (PRE), 20-30 min after cross-country jumping (XC), and 18-24 h after crosscountry jumping (POST) for horses competing in the CCI2* and CCI3* divisions of the 2006 Jersey fresh three-day event ${ }^{\mathrm{a}}$.

\begin{tabular}{lccr}
\hline Variable $^{\mathrm{b}}$ & PRE & XC & POST \\
\hline Plasma TP, g/dL & $7.01 \pm 0.06^{\mathrm{x}}$ & $7.31 \pm 0.07^{\mathrm{y}}$ & $6.76 \pm 0.07^{\mathrm{z}}$ \\
Plasma CORT, ug/dL & $5.47 \pm 0.43^{\mathrm{x}}$ & $11.1 \pm 0.70^{\mathrm{y}}$ & $7.55 \pm 0.36^{\mathrm{z}}$ \\
Plasma AST, U/L & $353.2 \pm 33.8^{\mathrm{x}}$ & $413.4 \pm 34.0^{\mathrm{y}}$ & $424.1 \pm 36.2^{\mathrm{y}}$ \\
Plasma LAC, $\mathrm{mmol} / \mathrm{L}$ & $0.82 \pm 0.04^{\mathrm{x}}$ & $11.2 \pm 1.60^{\mathrm{y}}$ & $0.62 \pm 0.03^{\mathrm{x}}$ \\
Plasma TOC, ug/mL & $5.50 \pm 0.28$ & $5.50 \pm 0.26$ & $5.75 \pm 0.33$ \\
Plasma RET, ng/mL & $163.4 \pm 4.6^{\mathrm{x}}$ & $177.0 \pm 5.7^{\mathrm{y}}$ & $152.9 \pm 4.4^{\mathrm{z}}$ \\
Plasma BC, ng/mL & $0.61 \pm 0.07^{\mathrm{x}}$ & $0.47 \pm 0.07^{\mathrm{y}}$ & $0.47 \pm 0.07^{\mathrm{y}}$ \\
RBC GPx, $\mathrm{mU}$ & $260.6 \pm 7.7^{\mathrm{x}}$ & $308.9 \pm 12.8^{\mathrm{y}}$ & $254.5 \pm 16.2^{\mathrm{x}}$ \\
\hline
\end{tabular}

${ }^{a}$ For each variable, no difference between divisions was observed; therefore, data was averaged across divisions.

bTP: plasma total protein; LAC: plasma lactate; CORT: cortisol; AST: plasma aspartate aminotransferase; TOC: plasma alpha-tocopherol; RET: plasma retinol; BC: plasma beta-carotene; GPx: erythrocyte glutathione peroxidase.

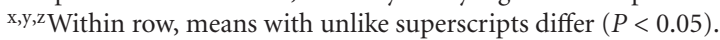


TABLE 4: Significant correlations between performance and antioxidant blood measures ${ }^{\mathrm{a}, \mathrm{b}}$.

\begin{tabular}{lcccccccccccc}
\hline Y & X & R & P & Y & X & R & P & Y & X & R \\
\hline BW & CORT & -0.21 & 0.02 & TP & GPx & 0.23 & 0.02 & CORT & GSH-T & 0.19 & 0.04 \\
BW & TP & -0.22 & 0.03 & TP & CORT & 0.25 & 0.002 & CORT & CK & 0.25 & 0.007 \\
Hct & RET & 0.22 & 0.03 & LAC & GSH-T & 0.52 & $<0.0001$ & RET & GSH-T & 0.40 & 0.01 \\
Hct & GSH-T & 0.66 & $<0.0001$ & LAC & GPx & 0.19 & 0.04 & TOC & BC & 0.40 & $<0.0001$ \\
Hct & GPx & 0.27 & 0.007 & LAC & CORT & 0.28 & 0.002 & TOC & AST & 0.30 & 0.002 \\
Hct & CORT & 0.44 & $<0.0001$ & LAC & RET & 0.27 & 0.004 & GSH-T & GPx & 0.28 & 0.003 \\
TP & CK & 0.25 & 0.01 & CORT & RET & 0.28 & 0.003 & GPx & AST & -0.19 & 0.04 \\
TP & GSH-T & 0.33 & 0.0007 & CORT & TOC & -0.19 & 0.04 & CK & AST & 0.50 & $<0.0001$ \\
\hline
\end{tabular}

${ }^{a}$ Horse was included in the model to test for significance, if insignificant, then it was removed from the model.

${ }^{\mathrm{b}}$ Initial BW $(n=112)$, hematocrit (Hct; $n=104$ total points), plasma total protein (TP; $\left.n=104\right)$, lactate (LAC; $\left.n=114\right)$, cortisol (CORT; $\left.n=114\right)$, alphatocopherol (TOC; $n=114)$, beta-carotene (BC; $n=114)$, retinol (RET; $n=114)$, serum creatine kinase (CK; $n=113)$, asparate aminotransferase (AST; $n=$ 113), erythrocyte total glutathione (GSH-T; $n=114)$, and glutathione peroxidase (GPx; $n=111)$.

\section{Discussion}

The main objective of this study was to determine the effects of a rigorous exercise activity, like a three-day event, on the antioxidant status of horses. To our knowledge, this is the first paper to reveal the antioxidant levels of horses competing in upper level divisions of a three-day event. A companion study studying inflammatory parameters and diet on a similar population of competitive horses was recently published [9]. Overall, our findings suggest that the cross-country jumping phase of a three-day event was a rigorous acute exercise bout that challenged the antioxidant systems of the horse, with more of a challenge being placed on the CCI3* horses. Collectively, the competing horses had higher blood levels of RET, GPx, and GSH-T, but lower BC at XC compared to precompetition (PRE) indicating these antioxidants were mobilized to be used in a response to a challenge to the antioxidant system as a result of the crosscountry jumping phase. Specifically within divisions, the CCI3* horses had 2.5 times greater serum concentrations of CK, 1.5 times greater serum concentrations of AST, approximately 50\% lower plasma BC, and 70\% lower erythrocyte GSH-T, as compared to the CCI2* horses at XC. This might appear to contradict the above statement; however, it might also lead us to believe that the higher level CCI $3 *$ horses were more able to handle that level of competition and therefore did not need as much GSH mobilized.

4.1. Time Effects. We found that GSH-T increased at XC in both divisions but decreased below baseline after 18 to $24 \mathrm{hr}$ of recovery. One recent study did look at antioxidant status in horses during a moderate level jumping competition [10]. They found that nonprotein sulfhydryl groups (this group included glutathione) did not increase until $24 \mathrm{~h}$ after competition, but did theorize that the response was due to a compensatory reaction to the increase in pro-oxidants produced during exercise. Another study observed a decline in GSH (both total and reduced) within $15 \mathrm{~min}$ after a stepwise field exercise test of $7000 \mathrm{~m}$ in warmblood eventing horses [11]. The authors theorize the reason why the GSH$\mathrm{T}$ in the current study did not follow the same pattern as in previous studies could be due to the added stress of competition and jumping efforts during competition in combination with galloping at approximately 9.2 to $9.4 \mathrm{~m} / \mathrm{s}$. The added stress creates a higher demand for the mobilization of GSH, hereby causing the increase after crosscountry followed by a compensatory decline during the recovery period of about $24 \mathrm{hrs}$.

A study involving endurance horses during a competitive race designed for research purposes evaluated antioxidant status with supplementation of vitamin $\mathrm{E}$ alone or in combination with vitamin C [7]. The $27 \%$ increase in RBC GPx observed in the last two stages of the $80 \mathrm{~km}$ race in both treatment groups likely reflected a response to utilize reduced glutathione during the radical scavenging process (reduced glutathione donates an electron to reduce a wide variety of hydroperoxides using GPx as a catalyst). It also reflected the consumption of pro-oxidants generated during exercise. This same trend was found in the current study with GPx in horses competing in both divisions increasing about $18 \%$ over baseline. A similar increase was found with GSH$\mathrm{T}$ in both studies, with horses in our study increasing at XC $\left(\mathrm{CCI}^{*}=60 \%\right.$; $\left.\mathrm{CCI}^{*}=27 \%\right)$ and with endurance horses in the previous study peaking after the first $21 \mathrm{~km}$ and then slowly decreasing to below baseline by the recovery sample [7]. In general, blood and plasma GSH-T reflects recent fluctuations in these concentrations in muscle cells $[12,13]$. The typical pattern observed is an increase in concentration after the onset of exercise that continues for a period of time during recovery then drops below baseline by about 18 to $24 \mathrm{hr}$. This pattern was observed here and in previous studies of horses engaged in intense and endurance exercise $[2,5,14]$.

Previous studies on the eventing horse have focused more on performance measures, including plasma LAC and CORT, serum CK, AST, and ion and water loss, rather than on antioxidant status [15-19]. This exercise challenge did elicit many other biochemical changes similar to that observed in other studies on the event horse. These measures are indicators of the rigor or intensity of the exercise being performed. They also help the researcher determine the level of fitness of the horse. Linden et al. [15] found similar blood concentrations as found in the current study of Hct, $\mathrm{TP}$, and LAC in horses before and after the cross-country 
phase of a low level event (similar to a CCI ${ }^{*}$ ). The CORT levels found in the current study were about 40 to $70 \%$ higher than what was observed in the previous study [15]. The similar LAC concentrations indicate the horses are probably more fit and able to handle the higher intensity of exercise than the lower level of exercise in the previous study. However, the higher CORT concentrations observed here may be due to the fact that our study was conducted on horses exposed to a more rigorous acute exercise and a higher level of competition than in the prior study. This higher concentration of CORT may be indicative of more physiological stress placed on the horses during this high level of competition. This can also be seen with a recently published study using horses subjected to a lower level of jumping competition, where the postexercise sample had two to three times less LAC, CK, and AST concentrations than the CCI3* horses in the current study [10].

4.2. Division Effects. Several studies have investigated horses competing at different levels during three-day events and found varying results in terms of heart rate, temperature, LAC, CK, and AST responses [17-20]. One study investigated $\mathrm{CCI}^{*}$ and $\mathrm{CCI} 2{ }^{*}$ horses competing in the long-format version of three-day eventing including roads, tracks and steeplechase phases during the same day of competition as the cross-country jumping phase [19]. The LAC, CK, and AST concentrations were similar between divisions, which the authors stated was not expected due to the considerable differences in speeds and distances of the two divisions. The LAC results after cross-country jumping were higher in the current study, despite the samples before exercise being similar in both studies. This could be due to the additional phases needed to complete before cross-country jumping. The current study did not find differences in LAC or AST concentrations between the $\mathrm{CCI} 2 *$ and ${ }^{*} \mathrm{CI} 3{ }^{*}$ divisions, similar to what was found previously, probably due to the degree of fitness and athletic ability of the higher level horses. As for the CK in the previous study [19], there were no differences found between divisions, which does not agree with the results found in the current study; the $\mathrm{CCI}^{*}{ }^{*}$ horses had higher levels of the enzyme after crosscountry jumping (2.5 times higher CK than PRE). Also AST was 1.5 times higher numerically with only a trend for a division difference $(P=0.07)$. However, the numerical values were similar between studies. No recovery samples were taken in the previous study [19], so no comparisons can be made; however, it is important to point out that the AST concentrations remained higher in the CCI3* horses 18 to $24 \mathrm{hr}$ after cross-country jumping. Another study looking at $\mathrm{CCI} 2 *$ horses competing either with or without roads, tracks and steeplechase also found no differences between these divisions for LAC, CK, AST, and other electrolyte measures [20]. Overall, these physiological measures are markers of exercise intensity and muscular effort during the exercise; however, they vary with horses physical fitness, age, gender, season of the year, and so forth. The current study shows that these subjects have fallen within wide variation of normal ranges for exercise at this level of intensity despite the differences between divisions.
Division effects were also found with GSH-T. One theory to explain the higher concentration in the CCI2* horses after cross-country jumping is that they were less conditioned or physically fit in order to handle that level of exercise stress compared to $\mathrm{CCI} 3 *$ horses therefore required higher concentrations of antioxidants. However, the $\mathrm{CK}$ response was lower for the $\mathrm{CCI} 2 *$ horses, indicating that $\mathrm{CCI} 2 *$ course placed a lower demand on the muscle cells of those horses compared to $\mathrm{CCI} 3 *$ horses. In a study looking at endurance races of varying intensity, there was higher GSH-T in the horses competing in the more intense endurance race, which is the opposite of what was found in the current study [21]. However, the endurance races did take place during different years and had drastically different environmental conditions; the more intense race had hotter and more humid ambient conditions could also have contributed to the higher GSH-T concentrations. The horses competing in the more intense endurance race also had higher CK concentrations, which reflect what we observed in the present study. Another theory for the higher concentrations of GSH-T in the CCI2* horses as compared to the $\mathrm{CCI} 3{ }^{*}$ horses could also be due to dietary effects. Diet plays an important role in antioxidant status in humans as well as horses. As mentioned below in more detail, the CCI $2 *$ horses were found to be receiving more antioxidant supplements and had longer access to fresh green pasture (number one source for antioxidants in horses) than the CCI3* horses. Whether these amounts are enough to definitely increase antioxidant status is unknown, but it is a possibility given the current results.

4.3. Correlations. A positive correlation between serum AST and plasma TOC, like that observed in this study, supports the hypothesis that free radicals produced during exercise change membrane permeability of muscle cells [1]. This same correlation has been observed in previous studies looking at the effects of endurance competition on horses $[21,22]$; however, the current study only found TOC to be correlated (positively) with AST. Even though most of the correlations presented could be used to infer that measures of oxidative stress and antioxidant status are a poor predictor of muscle enzyme leakage because it only explains a small amount of the variation, the authors feel that physiologically there was enough relevance to warrant the discussion.

Plasma LAC correlated positively with GSH-T, GPx, RET and CORT, which can be explained by the fact that all these measures peaked at $\mathrm{XC}$ and returned or was lower at POST. As for CORT, we found a positive correlation with RET, GSH-T, and CK, which again all increase with exercise, but a negative correlation with TOC. This negative correlation is a little harder to explain physiologically. Due to structural cellular changes during exercise like the increase in hematocrit due to splenic contraction and expulsion of erythrocytes in horses and an increase in muscular mitochondria that uptake additional vitamin $\mathrm{E}$, any change in vitamin E plasma concentrations remains constant because the production anddegradation are overshadowed by the redistribution. The current study did not have an effect of exercise on plasma TOC, which is further evidence against the belief that TOC is released during lipolysis associate with 
exercise. Other studies in human subjects have never been shown to be true and leave vitamin E bioavailability factors not well defined [23]. When taking into account the direction of responses, it makes more sense due to the fact that TOC does not increase at the XC sample and return to baseline or lower by POST as with the other measures as mentioned above.

Correlations with GPx and AST in the current study were negative, which is similar to one of the endurance competitions compared in a previous study [21]. However, the previous study in endurance horses found a positive correlation with a second endurance competition compared. These differences in the previous study were thought to be due to the difference in ambient conditions, terrain of the competition and level of difficulty. The present study showed a correlation similar to that of the more intense endurance competition in the previous study [21]. That endurance race was also an early season competition, with more horses that may not have been fully conditioned for the exercise required by the race. This could have been similar to the competition in the present study, which took place in the spring. Explanation of each correlation is difficult due to the transient nature of each marker and the amount of variability in each though worth reporting to share the comparative aspect of the results.

4.4. Diet Effects. Because feeding management of horses could not be controlled prior to competition, the effects of diet on antioxidant status could not be evaluated. However, a nutritional survey was conducted with the results summarized and reported elsewhere [8]. As mentioned earlier, the two most notable findings that could have possibly influenced antioxidant status of horses were the differences in hours of pasture turnout prior to arriving at the competition venue and antioxidant supplement use between $\mathrm{CCI} 2 *$ and $\mathrm{CCI} 3 *$ horses. In the current study, CCI2* horses were turned out for more hours each day prior to the competition compared to CCI3* horses $(12.3 \pm 1.3$ versus $9.1 \pm 1.3 \mathrm{hr}$, resp.). Since pasture is a good source of beta-carotene, the precursor to vitamin A that may be an explanation for why the CCI2* horses had higher BC levels before and after cross-country jumping. Another notable finding was that horses in both divisions received an average of 4 supplements per day and that more CCI ${ }^{*}$ horses received supplements specifically formulated as antioxidant supplements as compared to CCI3* horses $(42.1 \%$ versus $8.7 \%$, resp.). Antioxidant supplements fed typically contained vitamins $\mathrm{E}, \mathrm{C}$, and selenium, which, due to the antioxidant interaction and recycling, could partially explain the higher concentrations GSH-T in the CCI2* horses as compared to the CCI3* horses. Despite the difference in antioxidant supplements, we did not observe a difference in the number of $\mathrm{CCI} 2^{*}$ or $\mathrm{CCI} 3^{*}$ horses fed multi-vitamin and mineral supplements $(21.0 \%$ versus $21.7 \%$, resp.) or all-in-one supplements (5.3\% versus $8.7 \%$, resp.), which often contain vitamin E, C, and selenium as well. Given the horses' good BCS, sound feeding management programs, and supplement administration, it is likely that many of the horses were consuming antioxidants in adequate amounts to combat the oxidative stress experienced as a result of the three-day event. However, if specific recommendations for antioxidant supplementation for three-day event horses were to be developed, the antioxidants fed to the CCI2* horses should be investigated further.

\section{Conclusions}

In conclusion, the cross-country jumping phase of a threeday event is a challenge to the horse's antioxidant systems, with the $\mathrm{CCI} 3 *$ competitions being a more rigorous exercise bout than the CCI2*. The increase or upregulation of markers of antioxidant status is potentially due to increased scavenging of ROS created by oxidative stress experienced by horses during intense exercise. The ROS could also cause increased muscle membrane permeability as observed by the increase in muscle enzyme concentrations in the blood. Horses that are more fit may be better able to handle the stress of a CCI3* three-day competition, but the increased intensity also places a great deal of stress on the muscle cells more than in lower levels of competition. It is also likely that levels of antioxidants in the diet could enhance the horse's ability to cope with the physical demands of the crosscountry jumping phase of a three-day event. The question now becomes how much oxidative stress is too much and how much supplementation is really necessary to combat this stress or if by combating the stress we are disturbing the natural adaptation mechanisms necessary to allow the body to cope with physical and psychological stresses.

\section{Acknowledgments}

The authors thank the Jersey Fresh event organizers, J. Cory and L. Mathews, and Technical Delegate, R. Muller, for making this study possible. They also thank the FEI head veterinarian, D. Williamson, DVM., the competitors who allowed us to collect data on their horses during the competition, and the volunteers who helped with data collection. Many thanks are due to Laura Gladney, Program Associate, Rutgers University for her edits.

\section{References}

[1] J. M. McBride and W. J. Kraemer, "Free radicals, exercise, and antioxidants," Journal of Strength and Conditioning Research, vol. 13, no. 2, pp. 175-183, 1999.

[2] E. Chiaradia, L. Avellini, F. Rueca et al., "Physical exercise, oxidative stress and muscle damage in racehorses," Comparative Biochemistry and Physiology B, vol. 119, no. 4, pp. 833-836, 1998.

[3] A. White, M. Estrada, K. Walker et al., "Role of exercise and ascorbate on plasma antioxidant capacity in thoroughbred race horses," Comparative Biochemistry and Physiology A, vol. 128, no. 1, pp. 99-104, 2001.

[4] A. Frankiewicz-Jóźko and E. Szarska, "Anti-oxidant level to exercise in the blood of endurance horses," Biology of Sport, vol. 17, no. 3, pp. 217-227, 2000. 
[5] D. J. Marlin, K. Fenn, N. Smith et al., "Changes in circulatory antioxidant status in horses during prolonged exercise," Journal of Nutrition, vol. 132, no. 6, pp. 1622S-1627S, 2002.

[6] D. R. Henneke, G. D. Potter, J. L. Kreider, and B. F. Yeates, "Relationship between condition score, physical measurements and body fat percentage in mares," Equine Veterinary Journal, vol. 15, no. 4, pp. 371-372, 1983.

[7] C. A. Williams, D. S. Kronfeld, T. M. Hess et al., "Antioxidant supplementation and subsequent oxidative stress of horses during an 80-km endurance race," Journal of Animal Science, vol. 82, no. 2, pp. 588-594, 2004.

[8] A. O. Burk and C. A. Williams, "Feeding management practices and supplement use in top level event horses," Comparative Exercise Physiology, vol. 5, pp. 85-93, 2008.

[9] C. A. Williams and A. O. Burk, "Nutrient intake during an elite level three-day event competition is correlated to inflammatory markers and antioxidant status," Equine Veterinary Journal, vol. 42, no. 38, pp. 116-122, 2010.

[10] J. C. M. Soares, R. Zanella, C. Bondan et al., "Biochemical and antioxidant changes in plasma, serum, and erythrocytes of horses before and after a jumping competition," Journal of Equine Veterinary Science, vol. 31, no. 7, pp. 357-360, 2011.

[11] B. de Moffarts, N. Kirschvink, E. van Erck, T. Art, J. Pincemail, and P. Lekeux, "Assessment of the oxidant-antioxidant blood balance in a field exercise test in Standardbred and eventing horses," Equine and Comparative Exercise Physiology, vol. 2, pp. 253-261, 2006.

[12] T. D. Noakes, "Effect of exercise on serum enzyme activities in humans," Sports Medicine, vol. 4, no. 4, pp. 245-267, 1987.

[13] P. A. Harris, "Musculoskeletal disease," in Equine Internal Medicine, S. M. Reed and W. M. Baylye, Eds., pp. 375-397, WB Saunders, Philadelphia, Pa, USA, 1998.

[14] P. C. Mills, "Effects of exercise intensity and environmental stress on indices of oxidative stress and iron homeostasis during exercise in the horse," European Journal of Applied Physiology and Occupational Physiology, vol. 74, no. 1-2, pp. 60-66, 1996.

[15] A. Linden, T. Art, H. Amory, A. M. Massart, C. Burvenich, and P. Lekeux, "Quantitative buffy coat analysis related to adrenocortical function in horses during a three-day event competition," Zentralblatt fur Veterinarmedizin. Reihe A, vol. 38, no. 5, pp. 376-382, 1991.

[16] G. L. Ecker and M. I. Lindinger, "Water and ion losses during the cross-country phase of eventing," Equine veterinary journal. Supplement, no. 20, pp. 111-119, 1995.

[17] S. L. White, L. H. Williamson, P. L. Maykuth, S. P. Cole, F. M. Andrews, and D. R. Geiser, "Heart rate response and plasma lactate concentrations of horses competing in the cross-country phase of combined training events," Equine Veterinary Journal. Supplement, no. 20, pp. 47-51, 1995.

[18] S. L. White, L. H. Williamson, P. L. Maykuth, S. P. Cole, F. M. Andrews, and D. R. Geiser, "Heart rate response and plasma lactate concentrations of horses competing in the cross-country phase of combined training events," Equine Veterinary Journal. Supplement, no. 18, pp. 463-467, 1995.

[19] L. H. Williamson, F. M. Andrews, P. L. Maykuth, S. L. White, and E. M. Green, "Biochemical changes in three-day-event horses at the beginning, middle and end of Phase $\mathrm{C}$ and after Phase D," Equine veterinary journal. Supplement, no. 22, pp. 92-98, 1996.

[20] J. K. Murray, J. M. Senior, and E. R. Singer, "A comparison of cross-country recovery rates at CCI $2^{*}$ with and without steeplechase competitions," Equine veterinary journal. Supplement, no. 36, pp. 133-138, 2006.
[21] C. A. Williams, D. S. Kronfeld, T. M. Hess et al., "Comparison of oxidative stress and antioxidant status in endurance horses in three $80 \mathrm{~km}$ races," Equine and Comparative Exercise Physiology, vol. 2, pp. 153-157, 2005.

[22] C. A. Williams, D. S. Kronfeld, T. M. Hess, K. E. Saker, and P. A. Harris, "Lipoic acid and vitamin E supplementation to horses diminishes endurance exercise induced oxidative stress, muscle enzyme leakage, and apoptosis," in The Elite Race and Endurance Horse, A. Lindner, Ed., pp. 105-119, CESMAS, Oslo, Norway, 2004.

[23] M. G. Traber, "Vitamin E," in Modern Nutrition in Health and Disease, M. Shils, J. Olson, M. Shike, and A. C. Ross, Eds., pp. 347-362, Williams \& Wilkins, Baltimore, Md, USA, 9th edition, 1999. 


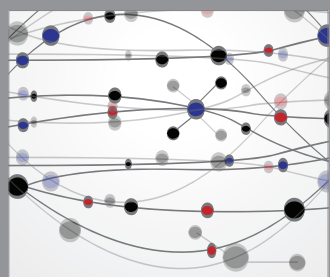

The Scientific World Journal
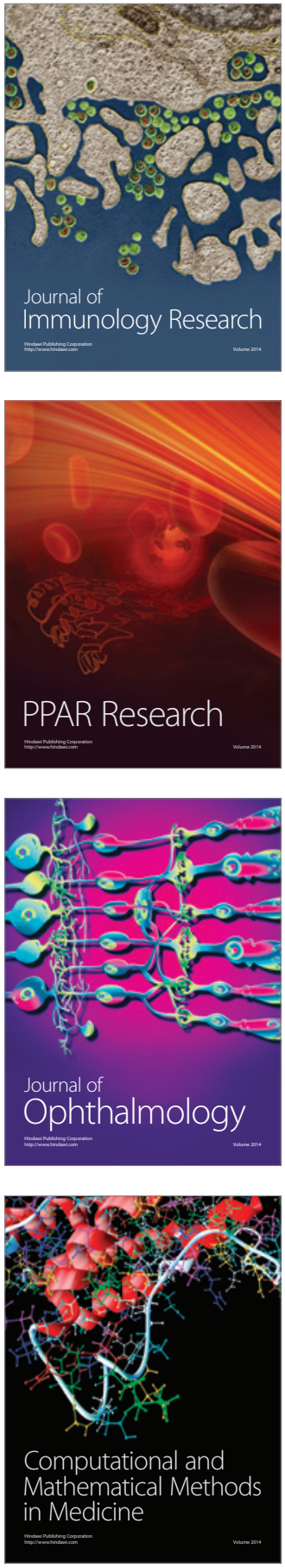

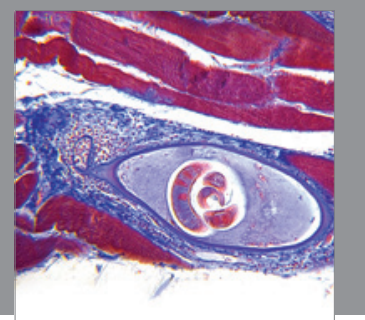

Gastroenterology

Research and Practice
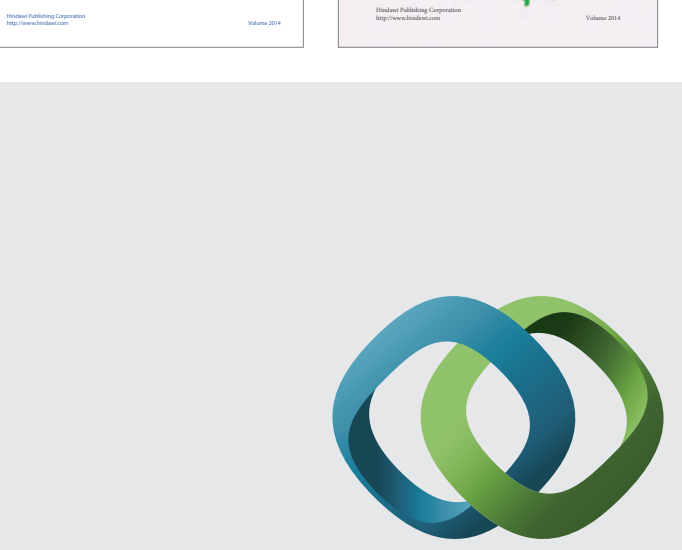

\section{Hindawi}

Submit your manuscripts at

http://www.hindawi.com
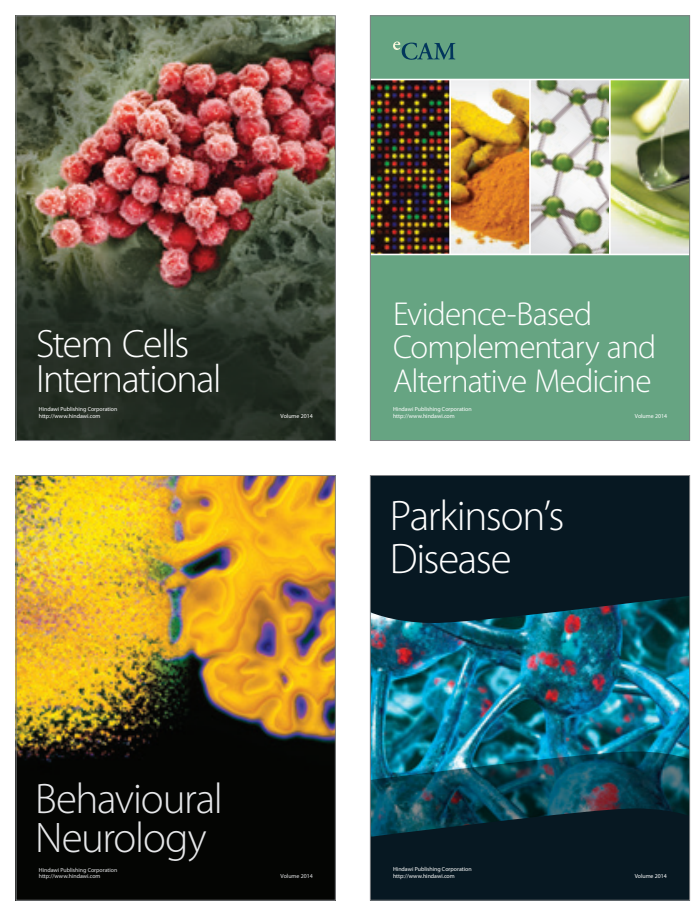

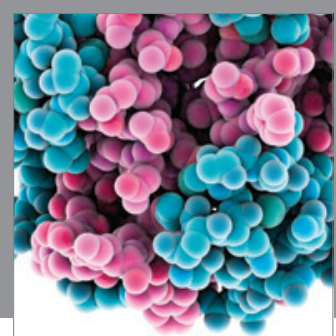

Journal of
Diabetes Research

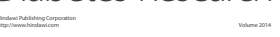

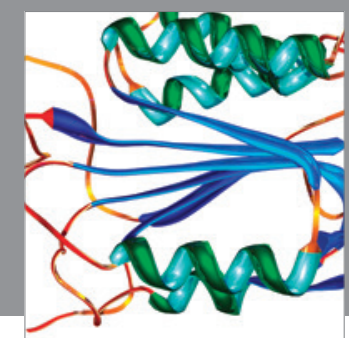

Disease Markers
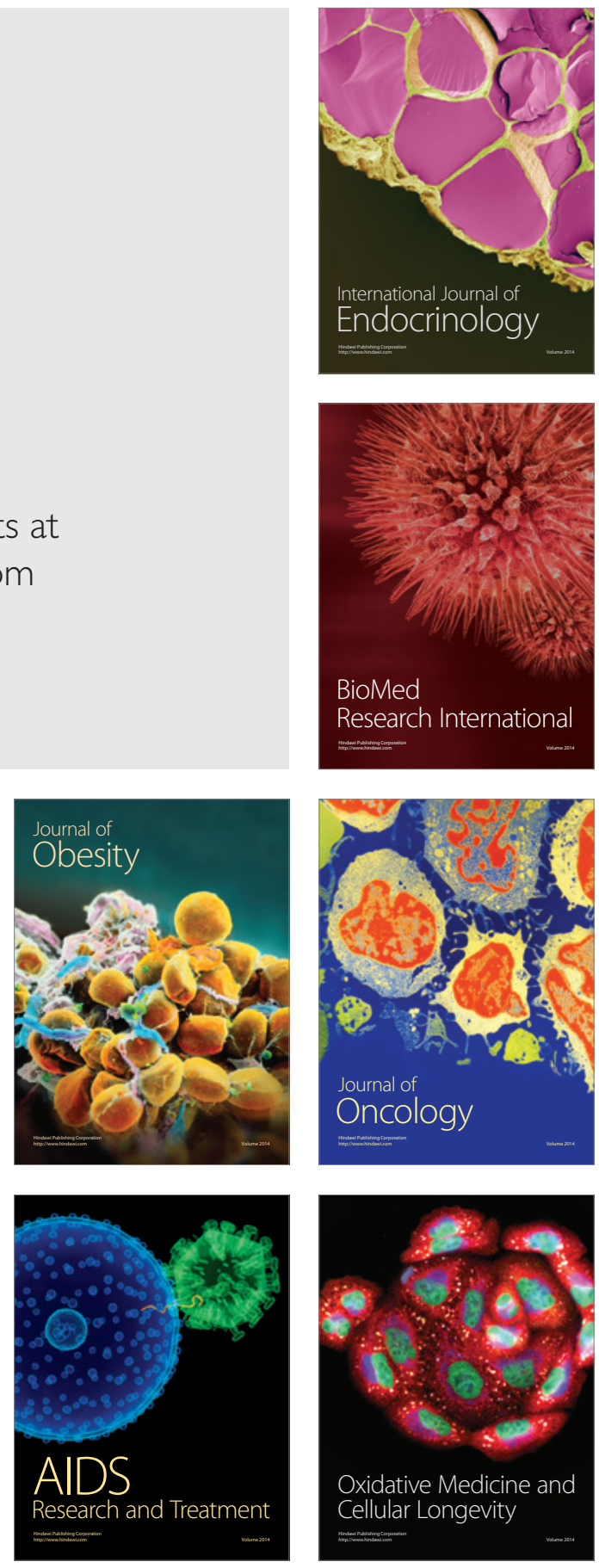